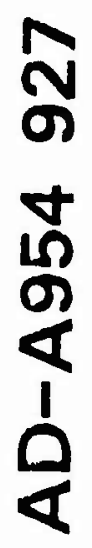

WATERTOWN ARSENA-

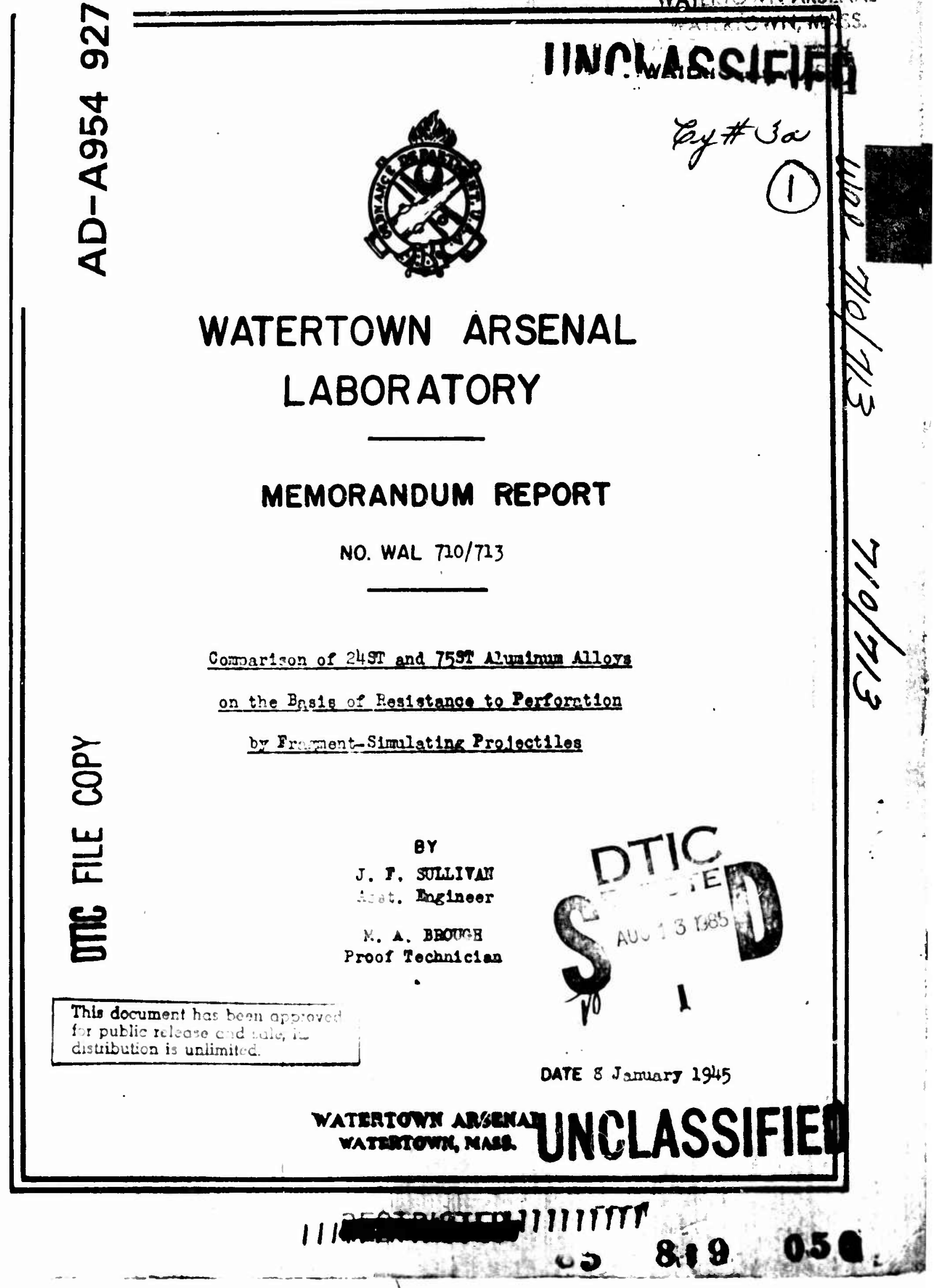



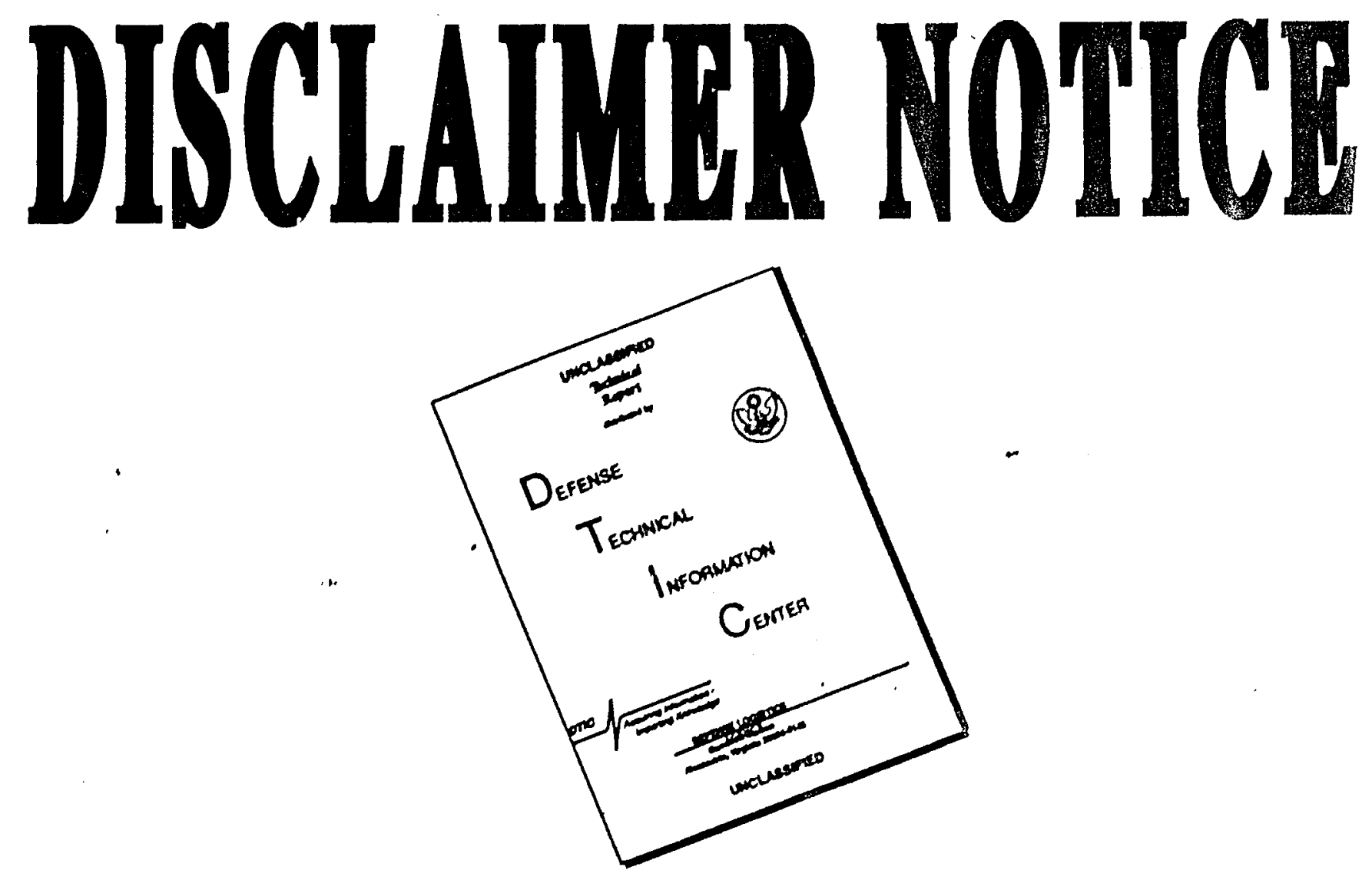

THIS DOCUMENT IS BEST QUALITY AVAILABLE. THE COPY FURNISHED TO DTIC CONTAINED A SIGNIFICANT NUMBER OF PAGES WHICH DO NOT REPRODUCE LEGIBLY. 
4. Although the resistance of individual pleces of 24 ST was sometimes higher than that of 75 ST it is felt that the results diaclose no signilcant difference between the resiatence characteristics of the two alloys under lapact of the project1les anployed in these tests. There 1s apparent, of course, the custosery inforlority of these materlal. to Hadfleld manganese steel of equivalent welght under these test conditions. It should be polnted out that this inferiority does not extrapolate to actual service conditions, bocause, under actual fragmentation teste (In which 20m. high oxplosive shell ere cetonated) the subject materials apnear to be superior to Hadfiald mancines otoel and It is considered that the latter tests are more signiftcant of actual service condition than ang ballistic limit tests currently in use.

APPRO TIS:

$$
\begin{aligned}
& \text { M10 Brough } \\
& \text { M. A. Brough } \\
& \text { Proof Techniclan } \\
& f y \text { Anitivan } \\
& \text { J. J. Sull1van } \\
& \text { As t. Instnoer }
\end{aligned}
$$

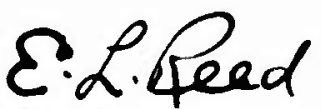

E. I. Reed

Research Motallureist

Acting Criaf, Armor Section 


\section{QABRT I}

Sumarr of Bellist10 Teste Conducted at Vetertown Argengl on Samples of 24 ST and 75 ST Duralumin Wh1ch Rad Boen

Previonsly Subjectod to Pramentation Tests

at Abordeen Proving Ground

\begin{tabular}{|c|c|c|c|c|c|c|c|c|}
\hline \multirow[b]{2}{*}{ Trpe } & \multirow{2}{*}{$\begin{array}{c}\text { Samplo } \\
\mathrm{No}_{0}\end{array}$} & \multirow{2}{*}{$\begin{array}{c}\text { Tominal } \\
\text { Gauge } \\
\end{array}$} & \multirow{2}{*}{$\begin{array}{l}\text { Actual } \\
\text { Gause } \\
\end{array}$} & \multirow{2}{*}{$\begin{array}{l}\text { Grans/ } \\
\mathrm{Sq}_{\mathrm{g}} \mathrm{Ft}_{\mathrm{e}}\end{array}$} & \multirow{2}{*}{$\begin{array}{l}\text { Equif. } \\
\text { Steol } \\
\text { Gange }\end{array}$} & \multicolumn{3}{|c|}{ Ballistic IInits } \\
\hline & & & & & & $.45^{1}$ & $Q_{-2}$ & a-1-s \\
\hline $75 \mathrm{ST}$ & 8 & .156 & $\begin{array}{l}.154^{n} \\
.154^{\prime}\end{array}$ & $\begin{array}{l}1026^{\prime \prime} \\
1008\end{array}$ & $\begin{array}{l}.055 \\
.054\end{array}$ & 922 & $960^{\text {the }} 25$ & $106 \div 23$ \\
\hline$*$ & 9 & .156 & .160 & 1043 & .056 & 847 & & \\
\hline * & n. & & .157 & 1028 & .056 & & $970 \pm 5$ & 107727 \\
\hline " & 2 & .156 & .156 & 1022 & .055 & 887 & & \\
\hline 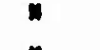 & 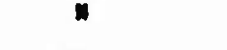 & & .155 & 2016 & .055 & & 95510 & $1083-23$ \\
\hline$"$ & 11 & .125 & .127 & 832 & .046 & 772 & & \\
\hline 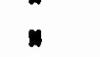 & 7 & 125 & .123 & $\begin{array}{l}798 \\
821\end{array}$ & $\begin{array}{r}.043 \\
.044\end{array}$ & 753 & $775^{-15}$ & $825^{1} 10$ \\
\hline 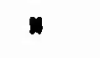 & $n$ & 0 & .125 & 816 & .044 & & $800 \pm 25$ & $803^{ \pm 23}$ \\
\hline 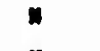 & 8 & .125 & $.12 \sqrt[4]{4}$ & 821 & .044 & 790 & & \\
\hline$*$ & * & & .127 & 838 & .045 & & $827 \pm 17$ & $898^{ \pm 13}$ \\
\hline " & 1 & .102 & .100 & 656 & .035 & 522 & & \\
\hline$n$ & * & 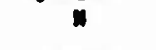 & .101 & 664 & .036 & & $728-13$ & $803-23$ \\
\hline$m$ & 4 & .102 & .103 & 672 & .036 & 537 & & \\
\hline$n$ & $n$ & $n$ & .102 & 674 & .036 & & $760 \pm 30$ & $720 \pm 25$ \\
\hline * & 20 & .102 & .102 & 665 & .036 & 542 & & \\
\hline$"$ & $n$ & & .102 & 662 & .036 & & $725+40$ & $723 \pm 13$ \\
\hline $245 T$ & $16-1-53$ & .156 & .156 & 1032 & .056 & 927 & 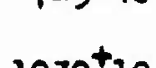 & \\
\hline$"$ & 7-1-69 & .156 & $\begin{array}{c}158 \\
161\end{array}$ & $\begin{array}{l}1028 \\
1046\end{array}$ & .050 & 880 & $1030-10$ & \\
\hline " & 1. & & .257 & 1022 & .055 & & 965 & $1042 \pm 17$ \\
\hline$*$ & $13-4-67$ & .156 & .157 & 1028 & .056 & 929 & & \\
\hline$n$ & & & .158 & 1032 & .056 & & $98 \div 23$ & $1090 \pm 15$ \\
\hline$\star$ & $10-B-179$ & .125 & .124 & 826 & .045 & 720 & & \\
\hline$*$ & $n$ & & .125 & 834 & .045 & & $825+27$ & $870 \pm 25$ \\
\hline " & $13-1-136$ & .125 & .224 & 837 & .045 & 777 & & \\
\hline 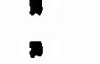 & 128182 & ת & .128 & 836 & .045 & $E \times 6$ & $825^{2}-10$ & $868-17$ \\
\hline - & $15-183$ & .125 & $\begin{array}{l}.127 \\
.126\end{array}$ & $\begin{array}{l}837 \\
836\end{array}$ & $\begin{array}{l}.045 \\
.045\end{array}$ & 936 & $835 \pm 20$ & $843+27$ \\
\hline$x$ & 16 & .102 & .104 & 684 & .037 & 568 & & \\
\hline " & $\sigma_{1}$ & & .105 & 678 & .037 & & $735+20$ & $710 \pm 20$ \\
\hline 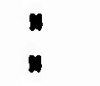 & 26 & .102 & $\begin{array}{l}.104 \\
.104\end{array}$ & $\begin{array}{l}685 \\
672\end{array}$ & $\begin{array}{l}.037 \\
.036\end{array}$ & 539 & $740+25$ & $690 t_{1}$ \\
\hline 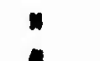 & 29 & .102 & .204 & 688 & .037 & 522 & & \\
\hline 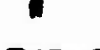 & & & .104 & 682 & .035 & $i$ & $77 \pm 25$ & $745+10$ \\
\hline$\frac{\text { rap }}{\text { Hadr! }}$ & $\frac{\text { PARISOE: }}{\text { d Kancancee }}$ & Steol & - & - & .045 & 950 & 1675 & 1050 \\
\hline & & & & & & & & \\
\hline
\end{tabular}

1. Cal..45 etenl-jackoted ball project11. - 230 gralne

2. Oel..22 Iracent-elmalating projectlle - 17 grain

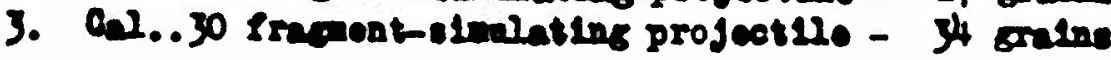

